\title{
On the Arrow of Time
}

\author{
Lucian M. Ionescu \\ Department of Mathematics, Illinois State University, IL 61790-4520, United States \\ Email: LMiones@ilstu.edu
}

\begin{abstract}
The interface between classical physics and quantum physics is explained from the point of view of Quantum Information Theory (Feynman Processes). The interpretation depends on a hefty sacrifice: the classical determinism or the arrow of time.

The wave-particle duality steams from the qubit model, as the root of creation and annihilation of possibilities. A few key experiments are briefly reviewed from the above perspective: quantum erasure, delayed-choice and wave-particle correlation.
\end{abstract}

Keywords: Space-time, quantum information, Feynman processes, quantum erasure

\section{Introduction}

Each unification of space, time and matter led to a new breakthrough: special relativity, general relativity. Yet the crucial progress was achieved when a unified description was provided for system observed and observer: quantum mechanics. As a next step, its universal time was relativistically upgraded in quantum field theory (QFT), and even a space-time duality was mathematically incorporated into conformal field theory.

The interpretation of quantum mechanics did not keep up with the mathematical-physics advancements (e.g. string theory in need of an interpretation, the measuring "paradox" with its quantum-classical divide etc.). The "... tension between quantum mechanics and space-time theory" [21], p.119, and many other conceptual difficulties are clear indications of "the need for a deeper physics" (loc. cit.). Instead, a lot of effort is consumed in trying to "save" the "old physics", by incorporating quantum mechanics (essentially) into a classical deterministic theory [10], p.10.

Fortunately all the pieces of the puzzle are ready to be set in place for a "big picture" resolving all the tension, provided we are willing to enlarge our "deterministic picture" as suggested by the mathematics of QFT; and the key concept at stake is (again) "Time".

In this article we will review briefly the quantum computing perspective on space, time and matter, as different aspects of Quantum Information Theory: data and programs $§ 2$ (for additional details see $[12,13,14])$.

Next we will point to the wave-particle interpretation of the qubit model of particle physics (Feynman Processes [16,17], with its precursor: spin networks and space-time foams §3. An interpretation "trick" disposes of the "collapse of the wave function" as entanglement of external observables.

The other main idea in this article consists in taking the CPT-Theorem at face value: the time arrow is a natural choice of orientation of our quantum computation; it's precursor is Feynman's interpretation of anti-matter as matter traveling backwards in time §4.

It is not easy to get comfortable with this interpretation, so we "practice" reinterpreting some typical quantum experiments (double slit, quantum erasure, delayed-choice etc.) §5.

In the concluding section we translate the consequences of our interpretation in a "less technical language", vulnerable to various speculations.

Additional explanations and an extensive bibliography can be found in $[13,14]$.

\section{On Space, Time, Matter and Information}

The benefits of adopting a computer science perspective on quantum physics are multiple; furthermore we advocate a "grand unification": Quantum Interactions are Quantum Communications. This prompts to the fact that space and time are mere coordinates in a quantum computation $[12,13,4]$, which could exist provided the quantum information flow is not "turbulent", as it will be explained bellow. 


\section{$2.1 \quad$ Space and Time}

Mathematically, this amounts to model a Feynman Process $[17,16]$ as a representation of a Feynman category, which is at least a hermitian category (left/right duality and compatible conjugation [2]). "Space" models the capability of parallel computation (tensor product $\otimes$ as "horizontal composition"), while "Time" models the capability of sequential computation (composition of morphisms as "vertical composition"). Both may be confined to a local existence, unless we disregard quantum corrections (loops/feedback etc.), which we view as "turbulent flow". In a representation of tangles for example, where Turaev's functor [24] is a simple instance of a Feynman rule, the input-output global computation can be factored in time-slices, but the information flow can move back relative to the global vertical coordinate which plays the role of time. The space-time structure is not preserved in such a quantum process, where space (external degrees of freedom DOFs) appear in pairs via products and coproducts (creation/annihilation).

\subsection{Consistent Histories}

A more general quantum computation (QC) of Feynman type, over graphs for example, could be factored as a network of subgraphs which include loops, in such a way that collapsing the quantum processes (viewing them as black boxes or "coupons" [24]), what remains is a representation of a POSET/distributive lattice conform to a classical computation compatible with our classical logic. This "picture" is in the author's opinion the essence of the consistent histories interpretation of quantum mechanics (see [23] for an introduction).

Within the quantum computations performed by the black-boxes (quantum chips) there is no admissible space-time coordinate system, yet the qubit-lines should be conserved (compare with quark-line diagrams).

The lack of a time coordinate, even locally, is reflected in theory as the need to specify an order product expansion in QFT, or the use of Keldish contour green functions etc.. It is also supported by experiments which are a clear indication of the failure of the classical model of a continuum space-time: the defiance of light speed in photon tunneling [1], p.10 and [7], p.50 (in our interpretation: random walk in time - see [14]), balanced homodyne detection [5], p.196 (noise interference which in our interpretation is due to quantum space-time fluctuations), entanglement and motion/simultaneity [10], p.10 (" ... a well-behaved time-ordering 't Hooft needs, simply doesn't exist: there is no causality at a deep level." ${ }^{1}$.

The two level hierarchy of the classical network (distributive lattice of cause-effect events of the consistent history model) of quantum processes, and responsible of the no clear-cut classical-quantum divide, is reminiscent of the equally not-so-clear-cut conscience-subconscience divide (see bellow §4).

\subsection{Matter}

What flows, i.e. "Matter", is quantum information, with its elementary constituent the qubit as an incarnation of the quantum non-exclusive dichotomy: "Yes and No". Symmetry, responsible for mass, and its generator entropy, is included as "program" acting on "data" [13,14].

A related quantum mechanics perspective on the information flow can be found in [4].

The role of the qubit as the elementary particle is proposed in [14]. Here we will focus on a "higher level" interpretation of the qubit.

\section{Particle-wave Duality and the Qubit Model}

The wave-particle duality is essentially the ability of classical models to explain some quantum phenomena, by switching between the wave model and particle model as needed (but not within the same experiment!).

The wave model is suited when new DOFs are created during the quantum experiment (e.g. alternative paths, entangled spins etc.), which subsequently entails interference. We consider the splitting/creation of new DOFs (external or internal) as the essence of the corresponding phenomenon, while the fringes of

\footnotetext{
${ }^{1}$ Causality is used here as of the "light-cone type", not in the sense of quantum correlations as we use it in Feynman Processes [17].
} 
interference, or merely the oscillatory dependence of the probability of detection, as consequences, well suited to be explained by a wave model (Schrodinger wave mechanics, de Broglie pilot-wave etc.).

The particle model captures localization of quantum events, as well as their distribution as quanta, suited to be counted (Heisenberg matrix mechanics as a dynamics of representations of quivers etc.). We consider the merger/destruction/collapse of DOFs as the essence of the corresponding phenomena, while the counting process is just a recording of this "collapse".

The creation and annihilation of DOFs is the trademark of QFT. We interpret the wave-particle duality as resulting from a predominance of one aspect over the other in typical experiments. There are though some experiments which involve both aspects $[22,3]$. The qubit model, where the unit of information

$$
\mathbf{H} \cong S U(2): \quad Q=z_{1}\left|Y>+z_{2}\right| N>, \quad z_{1}^{2}+z_{2}^{2}=1
$$

has both capabilities of splitting with interference and localization with counting (a number of qubit is defined), and therefore exhibits both aspects, of wave and particle.

The visibility $V$ as a measure of interference capability (wave) and the knowledge $K$ as a measure of (path) localization (particle) satisfy $V^{2}+K^{2} \leq 1$ [22], corresponding to the natural visualization of quaternions $\left(\mathbf{H} \cong S U_{2} \cong S^{3}\right.$ represented as two disks: projection of upper and lower hemisphere) [14].

The qubit $\left(S U_{2}\right)$ is essentially the "double binary entity" capable of splitting in "space" (parallel computing) and recombining in "time" (sequential computing) due to its structure:

$$
\mathbf{H}=\mathbf{R} \otimes\left(\mathbf{Z}_{2} \times \mathbf{Z}_{2}\right) / \sim
$$

The resulting model generalizes Heisenberg's picture (matrices as representations of quivers) and its continuum model QFT to Quantum Information Dynamics, where the emphasis is not on the amplitude of probability (S-matrix etc.) but on the actual flow of energy-matter/quantum information: Feynman Processes $[17,16,13,14]$.

The alternative incarnation is in the traditional Riemann surfaces, CFT etc., yet with the burden of continuity and ambient space-time which is a departure from the modern goal: to build a discrete theory of finite type (yet not finite), whether we believe the reality is (quantum) digital or not, since we need to discretize our model to do the computations and simulations on a computer anyway.

\section{CPT-Theorem Taken Seriously}

The concept of determinism is deeply rooted in science, culminating with the classical mechanics point of view. The determinism of quantum mechanics, in terms of deterministic amplitudes of probabilities was never enough to satisfy the urge for the "lost paradise" of classical physics, leading to periodic attempts to derive QM from a deterministic theory expressible in classical terms: space-time, trajectory etc. [13,10].

We propose an indirect way to "save" the determinism, with a hefty price though: sacrifice the arrow of time as an intrinsic concept ${ }^{2}$.

Recall the CPT-Theorem of QFT: a simultaneous reverse of parity, charge and time leaves the laws of physics unchanged. Let us take this theorem seriously, as if we really mean it ...

\subsection{Co-determinism}

As pointed out by Feynman, anti-matter (charge conjugation) can be interpreted as matter moving backwards in time. We extend this idea by claiming that, in some processes, quantum deterministic matter evolving forward relative to our time-arrow is classical deterministic anti-matter moving backwards in time. A bit more technically, the Feynman graph framework of QFT leads to composition of trees and cotrees ${ }^{3}$. This leads to the Hopf algebra framework of quantization and related aspects [14].

Intuitively, we, as cognitive processes define an arrow of time: "Cogito ergo sum". In our "quiet paradise" most other processes are "emitters" rather than "absorbers" of quantum information, except when deciding to set-up quantum experiments, exhibiting creation of internal structure; in this case we model quantum

\footnotetext{
${ }^{2}$ A victory a la Pirus!

${ }^{3}$ PROPs equivalent to products of operads and co-operads, as in a categorical Bohr's Law: $\mathcal{P}=\mathcal{O}^{*} \mathcal{O}[14]$.
} 
deterministically processes which are deterministic relative to the opposite time-orientation: our present meets their future before acknowledging their causes. The resulting model is QM (QFT) together with the collapse of the wave function. We in fact model possible outcomes backwards relative to their causality order and when we meet the "real cause" by measurement, we have to adjust our model.

The above interpretation is not just another interpretation of the same mathematical-physics generally accepted. It is conceptually compatible with (and even demanded by) the interpretation of elementary particle processes as quantum information flow (e.g. tangles, Feynman diagrams [18] etc. - see also [4]) forward as well as backwards relative to a local time-arrow (externally defined). We break the Input-Output symmetry (morphisms) because we distinguish between our "interior self" 4 and the "exterior world" from which we acquire information in a consistent manner enough to determine the Input-Output orientation of our mathematical-physics models.

\subsection{Matter-Antimatter}

As a surprising "side-effect", this also explains the "matter-antimatter "paradox": Why do we see only "matter"? where is the "anti-matter", if the "Big-bang" was symmetrical etc.

The "answer" (in the context of the above interpretation) is: it is "here", moving backwards in time. Then there should be "almost" the same amount of quantum information moving deterministic along our knowledge journey through the universe as well as quantum information moving co-deterministic, which we "swim" against in the "ocean of information" (tensor category with duality and hermitian conjugation etc.). If we compare the mass as a quantity of quantum information (or the equivalent amount of energy) with the usual communication streams (in the order of terabytes probably), we see that the universe is "almost symmetrical" relative to the matter-antimatter inversion.

\subsection{Collapse of the Wave Function}

The co-deterministic picture just decrees that the collapse of the wave function is legitim, when the dynamics is dualised and modeled using coproducts and superpositions. There is no absolute determinism; the classical determinism ("particles/ products/ functions") together with the quantum determinism ("waves/ coproducts/ trees") can be restated as a generalized determinism, relative to the "local timearrow".

This is even better: reality altogether is deterministic, yet we cannot know everything to predict "everything", and the future will always surprise us; that's what the free will is for!

\section{The Quantum-Classical Interface: Theory and Examples}

In this section we "test" our interpretation on some characteristic quantum processes.

\subsection{Quantum Erasure}

The quantum erasure experiment can be reproduced at home [6] (see also $[9,25])$, or at least the double slit quantum interference [19]. It consists in manipulating the proportion of the "wave model" and "particle model" within the same experiment, in what appears to be a temporal succession relative to observer's global time and arrow.

The first part of the experiment consists in a classical double slit experiment, and we will talk about electrons being sent to the double slit screen and then scattering photons from a 2nd source, to distinguish easily between the various qubits involved.

If the double-slit experiment is concluded without further interaction with the ambient/prepared environment, it yields the well-known fringes of interference ${ }^{5}$.

In this case the interpretation "tilts" towards a wave behavior: the electron "takes" both paths and corresponding to the differences in length, "part A" of the electron, which "went" through one opening

\footnotetext{
4 ... that we don't know enough of: "Nosce te ipsum".

${ }^{5}$ Bigger than expected by the author at least: [19].
} 
interferes with "part B" of the electron, which "went" through the other slit (one can in principle deem the source of electrons to produce an "electron at a time").

Now comes the observation (measurement/interaction) by "shining" a 2nd light source (not coherent with the 1st source) at the double slit panel. When an interaction occurs between a "part" of an electron and a (...!?) photon, the "which-way information" becomes available.

In the cited references [6] the authors make the usual assumption: observing the electron at one slit implies it went only through that slit. "If we measure which of the two ways the particle went," (loc. cit.) "we will only ever measure that it went exactly one of those ways.".

Their statement amounts to a reduction of the wave-particle model of the electron to a particle model once the measurement (observation of the incoming electron) was made, justified by the fact that the interference capability of the electron is "gone". Here it is only at the level of the interpretation/modeling, that the "collapse" from a wave to a particle model occurs; in fact we don't know if the electron did not go through the other slit too, sneaking unnoticed (not measured) to the final measuring site (as it will be proposed shortly)!

Reiterating, they assume that "seeing" the photon at the A-slit excludes an interaction at the B-slit (classical XOR). This is a localization made at the level of the interpretation, i.e. converting it to a particle model.

Now, why admitting that the electron can split like a wave when interference is recorded at the target, but deny this possibility (wave behavior) to the second particle, the photon, when it comes from the 2 nd source? In other words, why modeling the second photon as a particle only, and not as a wave-particle?

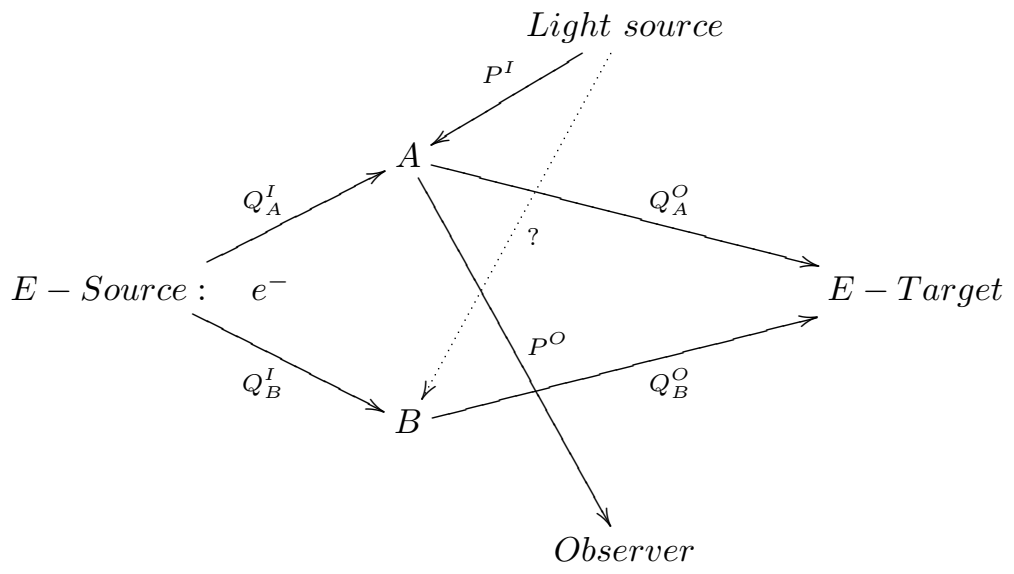

The quantum deterministic interpretation We propose a quantum deterministic interpretation ${ }^{6}$ which keeps the wave-particle duality, using the qubit model, explaining in a different way the lack of interference ("decoherence" in some sense), but in this way maintaining the capability for interference to explain the restoration of interference by quantum erasure.

Specifically, the double slit acts as a quantum beam splitter, which produces a pair of entangled qubits (a non-localized electron/wave character). When they are measured at the final target $T$, they interfere producing the fringes of interference (a quantitative investigation will appear elsewhere). When the 2nd source produces a photon/ (one qubit for now) not coherent with the electron, an interaction with the qubit $A$ represents a measurement which reduces the entangled state, implying that the other qubit $B$ has "opposite amplitude of probability". In essence, the entanglement of internal DOFs, like spin, is extended to external DOFs (paths), with the usual flavor of "spooky action at a distance".

At this point we mention the alternative to be explored: the interaction changes randomly the phase of $Q_{A}$, since the two sources are not synchronized ${ }^{7}$, and therefore it still does interferes with $Q_{B}$, but randomly, so the interference pattern is lost. The scattered photon is entangled with qubit $Q_{A}$, so the "scrambled" phase can be restored sending the information carried by the photon to the measurement site, to restore the interference pattern.

\footnotetext{
${ }^{6}$ Later we will inspect the alternative classical codeterministic explanation.

${ }^{7}$ Synchronized events: "same time=same space-like reference frame" $\cong$ "same phase" (compare with Einstein's analysis of "time").
} 
Now taking seriously the qubit model, we split the photon ("the particle") into two qubits $P_{A}$ and $P_{B}$ as being also a "wave-particle", corresponding to the two slits viewed from the other side.

The electron-photon interaction at the two slits subsystem results in the entanglement of external and internal information, i.e. the which-way and the internal phase information, of the two entangled qubits of the electron with the two qubits of the photon (quiver isomorphic to the homotopy class of a QFT Feynman diagram [15]).

In this way we avoid using the classical logic underlying statements like "a particle have traveled one way (X)OR the other" when explaining the experiment.

In other experiments the polarization is entangled with the which-way information. In fact, as mentioned before, splitting a qubit amounts to entangling the which-way information with itself.

Entangling the external/internal DOFs and then "erasing" (the which-way information) is implemented at the level of qubits by the rotations in the external basis or internal $\mathbf{C}$-space corresponding to the complex structures (hyper-Kahler) of the qubits (quaternions/Pauli matrices - see [14].

Regarding the erasure part, it seems to be a process of eavesdropping using light on the quantum communication using electrons:

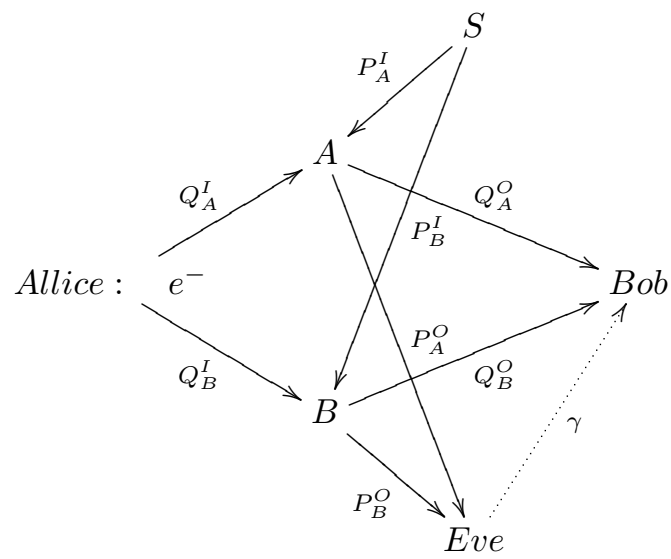

together with a teleportation achieving the erasure.

The eavesdropping reads part of the quantum information, which due to the no-cloning theorem, does not reach Bob anymore.

With no lens to focalize the two photon-qubits, their interference destroys part of the classical information needed to be sent to Bob to complete the teleportation of the "missing quantum information". If the lens is present, then the two qubits interfere constructively and sending the classical information completes the teleportation, allowing Bob to recover the interference (see teleportation and Bell measurements [26]).

The co-deterministic classical interpretation A two slit experiment can be equally viewed backwards relative to our time-arrow: an "anti"-photon from what we call "the target" (it's selfdual, but we would like to point out the CPT-aspects involved) is split, taking the two paths, interfering at what we call "the source" (Model the experiment in the opposite category). At this point the reader may object: "what if we consider a point on the target of destructive interference; there is no photon there!?? ${ }^{8}$ Let's assume there is dark matter! ${ }^{9}$. Then an "anti"-photon, white-matter or dark-matter (doesn't matter!) emerges from the target and takes one path to reach the source before it emerged, when measured relative to our time-arrow. Now if we look and see the photon at slit $A$, then it must have been white-matter, and it is "absorbed" by what we call the source $S \ldots{ }^{10}$

8 ... hm, right; but one can always adapt the theory!

9 A qubit with "opposite" amplitudes of Yes and No, canceling each other when reduced to an amplitude of probability.

10 This tentative "explanation" is left just in case the reader may do better applying the idea of co-determinism; the cases when it applies "in theory" are mentioned in the concluding section. 
Towards Infotronics The doubling of QFT from an "S-matrix theory" (C) to Quantum Information Theory as a dynamics of the qubit flow $\left(\mathbf{H}=S U_{2}\right)$ could also accommodate the dichotomy whitematter/dark-matter (like extending from the holomorphic to include the anti-holomorphic part).

Returning to [6] (the web-site supplement), the authors acknowledge that "Curiously, in the field of quantum computation, quantum physicists seem less hesitant to claim that the qubits are in a state of being " 0 " and "1" at the same time. Such statements are completely equivalent to saying that a particle passed through one slit and the other slit simultaneously."; quite right! We are claiming that, moreover, this is the case (i.e. results in a better model) independent of weather they have been measured or not!

In other words, at the modeling level, the possible transitions (paths) and interactions are the "reality" (space-time-matter), as far as we can "understand" from the corresponding observation (quantum communication).

\subsection{Delayed-choice Experiment and Wave-particle Correlation}

Similar interpretation assumptions ${ }^{11}$ occur in the delayed-choice experiments $[20,8,10]$ etc.

Initially proposed by Wheeler as a "gedanken experiment" [8], it was actually performed in various experimental arrangements (A. Zeilinger/[11], A. Suarez/[10], P. Aspect etc.).

Here again we advocate for the qubit model, quantum circuits [14], input-output ports, not just modulation of amplitudes of probability [8], against the assumption at the level of interpretation that "Thus the photon has traveled only one route" (loc. cit. p.967).

Wave-particle correlation experiments [22] rule out the naive wave-particle duality as a possibility to switch between the wave model and the particle model as needed, as if quantum mechanics is a "split extension" of wave mechanics and particle mechanics.

A quantitative explanation of visibility (interference) and which-way-information (localization) in terms of correlated/anti-correlated qubits will be given elsewhere.

\section{Conclusions}

In our opinion a comprehensive resolution of the conceptual tension in todays physics needs to address all the delicate issues, not just to focus on "this or that aspect". Therefore mixing mathematical-physics and "metaphysics" can hardly be avoided.

The first main idea is to focus on the qubit model ( $S U_{2}$-representations without ambient space-time) as primary when compared with the S-matrix approach. This determines us to claim "reality" for paths as well, and more generally, for transitions as "routes" taken by the quantum information when it splits into "packets", as the "best strategy" when a quantum communication is in progress in the World Quantum Web.

The qubit model implements the wave-particle duality: the wave characteristics come from a qubit representing space-like information (parallel processing) and the particle characteristics (counting/localized detection etc.) comes from the qubit's phase representing a local (Lie algebra/group: $T S^{1}=\mathbf{R}$ ) "entropy arrow" (input-output orientation). It is an alternative to the CFT approach using Riemann surfaces, delivering in this way the s/t-duality (mathematics level/ generalized Wick rotation [14]), a.k.a. waveparticle duality (physics interpretation).

The quantum erasure part will be explained quantitatively using the qubit model elsewhere, in the spirit of Infotronics: the quantum engineering of quantum computations on quantum circuits/networks [14].

The qubit model also pushes the quantum-classical divide to the "objects" (representing a collapse of DOFs; a clear-cut at the level of the model, but remember the resolution aspects when interpreting it) which model the sources and targets of the quantum transitions (the morphisms). "All qubits arrive" at the targets and interfere yielding a "click"/count etc., the discreteness and randomness of which is an indication of the non-existence of a local time, not even discrete.

The second main idea consists in pushing further the orthodox Feynman's interpretation of antiparticle as a particle traveling back in time, taking the CPT-Theorem at face value: the "not classically deterministic

\footnotetext{
${ }^{11}$ Choosing a model is unavoidably "Hypothesis fingo".
} 
matter" is anti-mater traveling backwards relative to our time-arrow determined by the Input-Output orientation of our quantum computation. At the level of the mathematical model, this amounts to considering the opposite (hermitian) category when interpreting portions of the consistent histories computation, with the amendment that morphisms should be enlarged as pairs of morphisms, in order to model relations ("functions and "cofunctions"; quasi-isomorphisms of PROPs).

Regarding the "time-reversal" interpretation, the experiments involving detection without interference (tree-like quiver) are classically co-deterministic, which appear relative to our time-arrow as quantum probabilistic (pure states plus collapse of the wave function). The experiments which involve "circuits", exhibiting wave and particle characteristics (in need of a combined wave-particle model, like the qubit model) are quantum deterministic processes, reversible, therefore also quantum co-deterministic.

The matter-antimatter "paradox" vanishes, as matter is also antimatter, and our breaking of symmetry of the "time" symmetry (in quantum processes where time exists, or at least can be organized as consistent histories) only alters the proportion slightly due to a natural choice of a time-arrow.

The measurement "paradox" seems to equally vanish if we accept that there is no absolute determinism, and the universe of quantum communications from which we are only a part, will always affect "our present" in a co-deterministic way.

The qubit model combined with the time-reversal idea seems to prompt for the dichotomy whitematter/dark-matter, consistent to the doubling process involved in the transition from an "S-matrix formalism" of QFT to the qubit flow of Quantum Information Theory and Infotronics.

As a side-effect of the mathematics needed by physics, it seems that a more symmetric treatment of classical mathematics is needed: functions and cofunctions, Hopf objects (for non-commutative geometry) instead of fields (commutative geometry, in need for quantization) etc. (see [14] for additional details).

The increasing use of discrete models (lattice models, graphs etc.) provides a short-cut from the classical science of Galileo-Newton-Einstein where limits were mandatory to smooth out computations, to the modern revival of the conceptual science ("Panta Rei", Zeno and discrete space and time etc.) freed-up of the necessary computational burden by computers as a counterpart to experimental technology as an interface to reality (Greeks never had their Galileo):

$\begin{array}{lccc}\text { "Reality" } & \text { Experiment } & \text { Interface } & \text { Theory } \\ \text { Classical } & \text { Galilei } & \text { Numerical Methods } & \text { Newton-Leibnitz-Einstein } \\ \text { Quantum } & \text { HEP\&LEP } & \text { Computer Simulations } & \text { Quantum MP\&CS. }\end{array}$

In conclusion, symmetry prevails and reassuringly, there is enough determinism making it worth planning ahead, with a (qu)bit of surprise to make use of our free will to chose our future depending on what it has in store for us.

\section{References}

1. M. Anderson, "Light seems to defy its own speed limit", New Scientist, 18 August 2007, p.10.

2. B. Bakalov, A. Kirillov, Lectures on tensor categories and modular functors, University Lecture Series, AMS Providence, Vol.21, 2001.

3. H. Carmichael, "Quantum fluctuations of light: a modern perspective on wave/particle duality", J. Evans and A. S. Thorndike eds., Quantum mechanics at the crossroads, The Frontier Collection, Springer, 2007, Ch.10, p.183-212.

4. B. Coecke, "Quantum information-flow, concretely, and axiomatically", quant-ph/0506132, Proc. SPIE 5833, Quantum Informatics 2004, 15 (July 28, 2005); doi:10.1117/12.620297.

5. J. Evans and A. S. Thorndike eds., Quantum mechanics at the crossroads, The Frontier Collection, Springer, 2007.

6. R. Hillmer and P. Kwiat, "A do-it-yourself quantum erasure", Scientific American, May 2007, p.90-95. (see also "What do the particles really do?", https://www.scientificamerican.com/article/quantum-eraser-what-do-quantum-particles-really-do/)

7. M. Holloway, "What visions in the dark of light", Scientific American, September 2007, p.50-53.

8. V. Jacques, et. al, "Experimental realization of Wheeler's Delayed-Choice Gedanken Experiment", Science 315, 966 (2007); DOI: 10.1126/science.1136303. 
9. P. G. Kwiat, B-G. Englert, "Quantum erasing the nature of reality or, perhaps, the reality of nature?", Science and Ultimate Reality: Quantum Theory, Cosmology, and Complexity, Edited by John D. Barrow, Paul C. W. Davies and Charles L. Harper, Jr.., Ch. 15, pp. 306-328, Cambridge University Press; http://research.physics.uiuc.edu/QI/photonics/sciam-supplemental.html

10. Z. Merali, "Entangled in the free will debate", New Scientist, 4 August 2007, p.10-11.

11. J. R. Minkel, "The Gedanken Experimenter", Scientific American, August 2007, p.94-96.

12. L. M. Ionescu, "The search of a new equivalence principle", âĂ̇̀, Scientific Journal International, Journal of Physical Sciences, Vol.1, No.1, 2007, pp.1-12 https://arxiv.org/abs/0705.1116.

13. L. M. Ionescu, The Digital World Theory v.1: An Invitation!, ISBN: 973-7744-39-x, Olimp Press, 2006; http://www.amazon.com/Digital-World-Theory-Lucian-Ionescu/dp/973774439X.

14. L. M. Ionescu, $Q++$ and a Non-Standard Model (DWT v.2), ISBN: 978-1-4251-3492-1; http://www.lulu.com/content/970826.

15. L. M. Ionescu, "Remarks on quantum physics and non-commutative geometry", Proceedings of the 19th National Philosophy Alliance Vol. 9, pp. 232-244; arXiv:math.HO/0006024, 2000.

16. L. M. Ionescu, "The Feynman Legacy", Int. J. Pure and Appl. Math., Vol.48, No.3, 2008, pp.333-355; arXiv:math/0701069.

17. L. M. Ionescu, "From operads and PROPs to Feynman processes", JP Alg. Number Theory and Applications, Vol. 7, No.2, pp.261-283, 2007; arXiv:math/0701299.

18. L. M. Ionescu, "Perturbative Quantum Field Theory and Configuration Space Integrals", Surveys in Mathematics and Its Applications, Vol.3 (2008), 79-110; arXiv:hep-th/0307062

19. L. M. Ionescu, "A quantum interference experiment", http://my.ilstu.edu/l̃miones/QE/experiments.html

20. P. Roussel, I. Stefan, "Is the interpretation of delayed-choice experiments misleading?", physics.pop$\mathrm{ph} / 0706.2596 \mathrm{v} 1$.

21. A. Shimony, "Aspects of Nonlocality in Quantum mechanics", [5] Ch. 6, p.107-123.

22. P. Schwindt, P. G. Kwiat, B-G Englert, "Quantitative wave-particle duality and nonerasing quantum erasure", Phys. Rev. A, Vol. 60, No.6, De. 1999, p.4285-4290.

23. A. Thorndike, "What are consistent histories", [5], Ch.8, pp. 149-157.

24. V. G. Turaev, Quantum invariants of knots and 3-manifolds. de Gruyter Studies in Mathematics, 18. Walter de Gruyter \& Co., Berlin, 1994.

25. A. Trifonov, G. Bjork, J. Soderholm, T. Tsegaye, "Comprehensive experimental test of quantum erasure", quant-ph/0009097.

26. W. K. Wooters, "Quantum entanglement as a resource for communication", [5], Ch.11, p.213-230. 